Both groups of children underwent a rapid step test, which has immediately confirmed the diagnosis, while the results of the microbiological culture came after 2-3 days.

It is noteworthy to mention that all children of the sample, have been treated in my paediatric practice.

Conclusion The comparative study confirmed that the rapid strep test is accurate in $97 \%$ of the examined cases. Namely, the parallel analyses consisting of rapid strep test and microbiological swabs in 269 children out of 380, have shown identical results.

Therefore, a rapid strep test is ought to be done by every doctor, in order to come up with a prompt diagnosis and therapy, prevent diseases, as well as promoting rational use of antibiotics.

\section{P400 QUALITY OF LIFE IN CHILDREN WITH INFLAMMATORY BOWEL DISEASE}

Anna Silonyan*, Andrey Surkov, Vladislav Chernikov, Elena Kulebina. National Medical Research Center for Children's Health, Moscow, Russian Federation

\subsection{6/archdischild-2019-epa.746}

Introduction Health-related quality of life (HRQOL) is an important indicator of the health status of children. In the Russian Federation in children with inflammatory bowel disease (IBD), this aspect has not been studied enough, but these data may help in the choice of tactics for maintaining patients.

Purpose of the study Rate HRQOL of children with IBD.

Participants and methods 54 children with IBD were examined: 28 children ( 12 boys and 16 girls) with Crohn's disease (CD) and 26 children (15 boys and 12 girls) with ulcerative colitis (UC). The median age was 15 years 4 months [13 years 1 month; 17 years 11 months]. The control group included 64 relatively healthy children, matched by age and sex. HRQOL was estimated using different age units of the children's form of the overall PedsQL questionnaire on a scale from 0 to 100 points, according to the following aspects: 'Physical Functioning' (PF), 'Emotional Functioning' (EF), 'Social Functioning' (SocF), 'School Functioning' (SchF),' Total Scale Score' (TS). To describe the data we used the median, 25th and 75th percentiles. Comparison of two independent groups by quantitative traits was carried out using the non-parametric method using the Mann - Whitney U-test.

Results When evaluating the indicators of HRQOL of patients with IBD and control group children, significant differences were obtained in the aspects of PF: 71.0 (58.25; $87.75)$ versus $87.0(78.75 ; 93.0)$ points, $\mathrm{p}<0.001$; EF: 65.0 $(50.0 ; 80.0)$ versus $75.0(61.25 ; 85.0), p=0.046 ;$ SchF: 60.0 $(45.0 ; 78.5)$ versus $75(60 ; 90.0), \mathrm{p}=0.004$ and TS: 69.57 $(58.7 ; 80.71)$ versus $82.0 \quad(73.25 ; 88.0), \mathrm{p}<0.001$. When comparing the responses of children with $\mathrm{CD}$ and UC, there were no significant differences in all scales of the questionnaire $(\mathrm{p}>0.05)$.

Findings The study found significant differences in the form of lowering HRQOL in children with IBD of relatively healthy peers in almost all aspects, which must be considered when managing patients with this chronic pathology. IDIOPATHIC ARTHRITIS DURING ETANERCEPT THERAPY

${ }^{1}$ Anna Fetisova, 1,2Ekaterina Alexeeva*, 1,2Tat'yana Dvoryakovskaya, 'Vladislav Chernikov ${ }^{1}$ Irina Vinyarskaya, ${ }^{1}$ Margarita Soloshenko, ${ }^{1}$ Rina Denisova, ${ }^{1}$ Kseniya Isaeva, ${ }^{1}$ Anna Mamutova. 'National Medical Research Center for Children's Health, Moscow, Russian Federation; ${ }^{2}$ Sechenov First Moscow State Medical University (Sechenov University), Moscow, Russian Federation

\subsection{6/archdischild-2019-epa.747}

Objectives To assess the influence of the genetically engineered biologic drug etanercept on the quality of life of patients with juvenile idiopathic arthritis (JIA).

Methods The research included 85 children with poly-and oligoarticular variant of the JIA at the age of $5(2 ; 17)$ years. The assessment of the quality of life of patients was carried out by means of the parental version of a special questionnaire CHAQ (Childhood Health Assessment Questionnaire), Health Utilities Index Mark 3 (HUI3) questionnaire. The doctor and parents of the patient also assessed the global activity of the illness by the $100-\mathrm{mm}$ visual analog scale (VAS) before the etanercept prescription and in 1, 6 and 12 months. All patients before the etanercept prescription received immunosuppressive agents.

Results Impressive increase in an average point of the quality of life (the response in 1 month after the therapy beginning) was noted by the attributes of a questionnaire of HUI3 «emotions» - from $0.82( \pm 0.18)$ to $0.90( \pm 0.13), \mathrm{p}=0.001$, «cognitive abilities» — from $0.85( \pm 0.17)$ to 0.91 ( \pm 0.18 ), $\mathrm{p}=0.006$, and «pain» — from 0.78 ( \pm 0.23) to 0.91 ( \pm $0.1), p<0.001$. In 12 months of the treatment the average point of the quality of life raised also by such attributes as «locomotivity» - from $0.85( \pm 0.3)$ to $0.99( \pm 0.06), \mathrm{p}<$ 0.0001 , and «fine motor skills» — from 0.9 ( \pm 0.19 ) to 0.98 $( \pm 0.05), \mathrm{p}<0.001$.

Conclusions Treatment with the etanercept provided the improvement of the quality of life of children with the JIA and their families, improvement of physical activity and emotional state of patients.

\section{P402 RECOGNISABLE PATTERNS TO CHILDHOOD EXPERIENCES OF MIND FORMATION}

Peter Kearney*. University College, Cork, Ireland

\subsection{6/archdischild-2019-epa.748}

The title deliberately echoes Smith's book on Recognizable Patterns of Human Malformation first published in 1970. Smith's patterns relate to antenatal events, whereas patterns that shape our minds relate to special experiences during childhood. As Paediatricians we are very familiar with incremental formation which we chart by measuring growth and evaluating development. We are less au fait with special experiences that shape our minds probably because Anthropology has been peripheral to medical education. The boundaries of different sciences are blurring as interest in the formation of our minds draws on many disciplines. Our deep interest in biological child health aims to treat and prevent diseases from the newborn to adolescence. Biology is not the whole story. The great status 